\title{
Balanceamento de fluxo ou balanceamento de capacidade? Análises e proposições sistêmicas
}

\section{Flow balancing or capacity balancing? Systemic analysis and propositions}

\author{
Diego Augusto de Jesus Pacheco ${ }^{1,2}$ \\ Daniel Pacheco Lacerda ${ }^{3,4}$ \\ Secundino Luis Henrique Corcini Neto $3,4,5$ \\ Carlos Fernando Jung ${ }^{2}$ \\ José Antônio Valle Antunes Júnior ${ }^{3}$
}

\begin{abstract}
Resumo: A melhoria no desempenho da manufatura pode ser alcançada por meio de diversas alternativas. Por um lado, a busca por elevados níveis de eficiência e utilização de todos os recursos através do balanceamento da capacidade de recursos produtivos, por exemplo, foi e ainda é uma prática comum nas empresas de manufatura. Por outro, há empresas que adotam em seu sistema produtivo as práticas de balanceamento de fluxo, a qual admite elevados níveis de eficiência e utilização apenas nos recursos restritivos do sistema, os gargalos. Frente a essas possibilidades, o principal objetivo do presente trabalho é discutir as diferenças e as implicações na manufatura entre realizar o balanceamento de fluxo e o balanceamento da capacidade. Conduziu-se a análise apresentando-se variáveis críticas à tomada dessa decisão gerencial. Para suportar o trabalho, fez-se a revisão bibliográfica pertinente ao tema e, em seguida, estruturou-se a discussão usando por base a abordagem do pensamento sistêmico. Essa abordagem, dentre outros aspectos, possibilita identificar relações de efeito-causa-efeito. Os resultados da investigação evidenciaram os pontos convergentes e divergentes entre as duas visões. Foi constatado que adotar decisões gerenciais considerando o balanceamento de fluxo combinado com determinadas práticas do balanceamento de capacidade pode contribuir para elevar o ganho global da empresa.
\end{abstract}

Palavras-chave: Decisão gerencial. Sistemas de manufatura. Balanceamento de fluxo. Balanceamento da capacidade.

\begin{abstract}
Manufacturing performance improvement can be achieved in several different ways. On the one hand, the search for high efficiency and maximized utilization in all system resources through balancing the capacity of productive resources, for example, is still a common practice in manufacturing companies. On the other hand, there are companies that adopt flow balancing practices in their production system, which promote high efficiency and utilization only in the restrictive resources of the system, the bottlenecks. Given these possibilities, the main objective of this paper is to evaluate the manufacturing implications and differences between flow balancing and capacity balancing. An analysis identifying the variables that are critical to this managerial decision making process was carried out. A literature review on this topic was conducted, and subsequently, the analysis was performed based on the System Thinking approach. This approach, among other things, allows the identification of effect-cause-effect relationships. The results obtained demonstrated points of convergence and divergence between these two views. It was found that adopting managerial decisions considering flow balancing combined with certain practices of capacity balancing can help increase the company overall gains.
\end{abstract}

Keywords: Managerial decision. Manufacturing systems. Flow balancing. Capacity balancing.

\footnotetext{
${ }^{1}$ Programa de Pós-graduação em Engenharia de Produção, Universidade Federal do Rio Grande do Sul - UFRGS, CEP 90035-190, Porto Alegre, RS, Brasil, e-mail: profdajp@gmail.com

${ }^{2}$ Curso de Engenharia de Produção, Fundação Educacional Encosta Inferior do Nordeste, Faculdades Integradas de Taquara - FACCAT, CEP 95600-000, Taquara, RS, Brasil, e-mail: carlosfernandojung@gmail.com

${ }^{3}$ Programa de Pós-graduação em Engenharia de Produção e Sistemas, Universidade do Vale do Rio dos Sinos - UNISINOS, CEP 93022-000, São Leopoldo, RS, Brasil, e-mail: dlacerda@unisinos.br, secundino@unisinos.br, junico@ produttare.com.br

${ }^{4}$ Grupo de Pesquisa em Modelagem para Aprendizagem - GMAP, Universidade do Vale do Rio dos Sinos - UNISINOS, CEP 93022-000, São Leopoldo, RS, Brasil

${ }_{5}^{5}$ Programa de Pós-graduação em Administração, Pontifícia Universidade Católica do Rio Grande do Sul - PUCRS, CEP 90619-900, Porto Alegre, RS, Brasil
} 


\section{Introdução}

São notáveis, sobretudo na manufatura, frequentes transformações nos sistemas produtivos em virtude do processo de globalização, da ameaça de novos concorrentes, da busca incessante da qualidade e redução de custos de produção como meio para incrementar o lucro e fortalecer a posição competitiva das empresas. Os meios para estabelecer esse fortalecimento variam em função das características de cada empresa, do segmento em que atuam, bem como dos produtos que comercializam (UMBLE; SRIKANTH, 1995). O impacto desse contexto no ambiente da manufatura se reflete na necessidade de alinhamento cada vez mais preciso entre a estratégia da organização e sua manufatura. Nesse sentido, Hayes e Wheelwright (1979), Slack, Chamber e Johnston (2009), Skinner (1969, 1974, 2007) e Miltenburg (2008) têm discutido a importância da manufatura como forma de sustentar a competitividade da empresa. Para Hayes e Wheelwright (1979), o sucesso da empresa é determinado pelo grau de alinhamento entre capacidade de mudança e a integração da operação com a estratégia da organização. Slack, Chamber e Johnston (2009) argumentam que construir e gerenciar ambientes de produção integrados é o maior desafio na manufatura. Essa integração visa estabelecer uma vantagem competitiva. Essa vantagem competitiva requer, não excludentemente, a busca pela melhor qualidade dos produtos, a oferta de serviços que propiciem soluções aos clientes e o baixo custo de produção.

Skinner (1969), quando investigou a manufatura americana, constatou que a maioria dos altos executivos e gestores percebia o sistema de produção da empresa com a noção de produtividade total e, por consequência, de eficiências dos recursos. A visão, à época, estava focada no custo reduzido, na alta qualidade e num nível de serviço aceitável pelo cliente. Segundo Skinner (1969), prevalecia, na amostragem estudada, a percepção de que a empresa manufatureira terá desempenho eficiente se possuir equipamentos modernos, sistemas informatizados e gestores cultos aliados a uma força de trabalho cooperativa. Todavia, esses fatores não seriam suficientes se a manufatura não servisse à empresa para alcançar suas metas estratégicas (SKINNER, 1969).

Tal debate ainda se mantém atual, à medida que outros trabalhos (GOLDRATT, 1992; COX; SCHLEIER JUNIOR, 2010) retratam que as ações locais (contextualizadas, nesse caso, na manufatura) devem estar subordinadas à busca pelo melhor resultado global (nesse caso, a empresa). O uso integrado da TOC, Lean e Seis Sigma para melhorar o desempenho da manufatura foi investigado em Pacheco (2014). Assim, para atender às metas da organização, uma decisão local que interfere no desempenho global (empresa) e prevalece no cotidiano da produção diz respeito ao planejamento da fábrica e ao adequado balanceamento de seus recursos e fluxos. Souza, Rentes e Agostinho (2002) e Ozpeynirci e Azizoglu (2009) relatam que alguns empresários e acadêmicos apregoam a alocação da carga dos recursos produtivos, indistintamente, baseada no atendimento à demanda solicitada pelo mercado. Assim, o balanceamento é função da capacidade desses recursos, estimulando o aumento de eficiência, visando a maximização das capacidades individuais.

Pacheco et al. (2013) propuseram um modelo de gestão da capacidade produtiva para sistemas de manufatura ao combinar os conceitos da Teoria das Restrições e da Total Productive Maintenance (TPM), usando o Índice do Rendimento Operacional Global (IROG) com uma abordagem diferente da que consta na literatura tradicional que trata do tema, apresentada por Nakajima (1988).

Seguindo nessa linha de discussão, autores como Souza, Rentes e Agostinho (2002), como Davis (1966), Hillier e Boling (1966), Payne, Slack e Wild (1972), El-Rayah (1979a), Goldratt (1992), Sarker (1984), Smunt e Perkins (1985), Fry e Russell (1993), Pike e Martin (1994), Chakravorty e Atwater (1996) vêm estudando maneiras de distribuir distintamente as cargas entre as operações. Essa distribuição consiste em um meio de melhorar a eficiência da produção como um todo e não somente de forma individual, nos postos de trabalho.

O efeito nocivo da busca pela alta utilização de todos os recursos na produção está relacionado com o aumento do inventário, do tempo de atravessamento (lead time) e da despesa operacional. Esses aspectos foram abordados por diversos trabalhos (UMBLE; SRIKANTH, 1995; GUERREIRO, 1995; ANTUNES JUNIOR, 1998; GOLDRATT; COX, 2002; JACOB; BERGLAND; COX, 2010) no campo de pesquisa da manufatura.

Dessa forma, parece pertinente, ainda, refletir a partir da seguinte questão: balancear o fluxo produtivo ou balancear a capacidade dos recursos? Portanto, o objetivo deste trabalho é discutir, a partir de uma perspectiva sistêmica, as diferenças e consequências para a manufatura entre a adoção de um ou outro desses critérios de balanceamento: fluxo e/ou capacidade.

A partir do contexto apresentado, o presente trabalho justifica-se ao somar-se aos esforços de pesquisa existentes abordando o tema a partir de uma perspectiva distinta. Aos gestores, a investigação é pertinente por contribuir no processo de tomada de decisão em manufatura, sobretudo quanto à definição de investimentos e ao planejamento da capacidade produtiva. Por sua vez, para a comunidade acadêmica, a contribuição faz-se na tentativa de fornecer uma relação dos principais aspectos das duas abordagens a partir de uma leitura sistêmica de suas relações e impactos. 
Na busca do objetivo traçado, esta pesquisa foi organizada a partir de um estudo bibliográfico (LITTELL; CORCORAN; PILLAI, 2008; GOUGH; OLIVER; THOMAS, 2012) sobre o tema que subsidiou a elaboração de um conjunto de estruturas sistêmicas. $\mathrm{Na}$ análise das estruturas sistêmicas foram identificados os distintos impactos para a empresa no utilização de uma ou outra abordagem.

Assim sendo, o trabalho está estruturado para, primeiramente, apresentar o referencial teórico acerca do balanceamento de fluxo e balanceamento de capacidade, seus pressupostos e limitações dentro de um sistema de produção. Em seguida é abordado conceitualmente o Pensamento Sistêmico e as vantagens do uso de tal abordagem para a análise qualitativa de situações complexas. Após isso, apresentam-se as estruturas sistêmicas: i) uma representando as relações envolvidas no critério do balanceamento de capacidade; ii) outra, as relações envolvidas no critério do balanceamento do fluxo. Nessa seção também são apresentadas as análises e discussões acerca do tema. Por fim, apresentam-se as considerações finais e sugestões de futuros trabalhos sobre o tema.

\section{Balanceamento de fluxo e balanceamento de capacidade}

Umble e Srikanth (1995) afirmam que é quase impossível promover o balanceamento efetivo da capacidade dos recursos em operações de manufatura. As causas que contribuem para isso são a maneira incremental de adição de capacidade, a influência da variabilidade e os eventos dependentes. As revisões da literatura realizadas por Ghosh e Gagnon (1989) e por Kriengkorakot e Pianthong (2007) apresentam contribuições importantes para a evolução do estudo desse tema. Dentre essas contribuições destaca-se a proposta de classificação dos sistemas produtivos em quatro categorias distintas:

a) Modelo único determinístico (Single Model Deterministic - SMD): um único produto é produzido no sistema, os tempos das atividades são conhecidos deterministicamente e o critério de eficiência é a otimização.

b) Modelo único estocástico (Single Model Stochastic-SMS): um único produto é produzido e introduz o conceito de variabilidade no tempo das tarefas, sendo mais realista em operações manuais onde os tempos de operação raramente são constantes.

c) Modelo de mix múltiplo determinístico (MultiMixed Model Deterministic-MMD): assume que os tempos das tarefas são determinísticos e introduz o conceito de um sistema produzindo múltiplos produtos. d) Modelo de mix múltiplo estocástico (Multi-Mixed Model Stochastic - MMS): múltiplos produtos são produzidos sob efeito da variabilidade, dificultando analisá-los. Frequentemente, o balanceamento é feito para cada modelo produzido no sistema.

As características dos modelos MMS e SMS, apresentadas em Ghosh e Gagnon (1989) e ratificadas por Becker e Scholl (2006), Scholl e Becker (2006), Kriengkorakot e Pianthong (2007) e Boysen, Fliedner e Scholl (2007), corroboram a visão de variabilidade e dependência na manufatura sugerida por Umble e Srikanth (1995). Portanto, essas pesquisas traduzem a realidade dos sistemas de manufaturas modernos voltados à produção de bens customizados, ou seja, submetidos à produção em pequenos lotes para compor os diversos produtos do portfólio comercial. Dentre os modelos apresentados, o MMS será o enfoque da discussão entre balanceamento de capacidade e balanceamento de fluxo realizada neste trabalho, uma vez que ele comporta a produção de um mix variável de produtos sob a influência da variabilidade.

A prática do balanceamento da capacidade remonta à produção em massa do início do século XX, onde a competição era baseada, essencialmente, nos baixos custos produtivos. Todavia, com a evolução dos mercados - geografia, tecnologia, diversidade de produtos etc. - novas dimensões competitivas passaram a ser exigidas das empresas, para que mantenham ou ampliem sua participação no mercado. Dentre as dimensões competitivas estão a flexibilidade, o desempenho nas entregas, a qualidade e a inovação. Uma análise detalhada sobre dimensões competitivas é encontrada nas obras de Hayes e Wheelwright (1979), Skinner (1969, 1974, 2007), Miltenburg (2008) e Slack, Chamber e Johnston (2009). Estudos recentes que avançam no debate das dimensões competitivas vêm sendo realizados. A análise do impacto do Lean Manufacturing, Seis Sigma e Teoria das Restrições em cada dimensão competitiva da estratégia de produção pode ser encontrada na ampla pesquisa realizada por Pacheco (2012). Os resultados da pesquisa feita pelo autor permitiram apresentar um modelo que a partir da estratégia de produção das Unidades de Negócio, integra a Teoria das Restrições, o Lean Manufacturing e o Seis Sigma, sugerindo uma lógica de priorização das suas práticas conforme as dimensões competitivas exigidas pelo mercado consumidor.

Camargo e Pacheco (2013), por exemplo, avaliaram o impacto no desempenho de um sistema produtivo da indústria calçadista, ao se aplicar as técnicas do balanceamento de linhas. O estudo permitiu reduzir e realocar a força de trabalho da linha, reduzindo os custos produtivos, a partir do remanejo de 18 pessoas.

Outro elemento contributivo com enfoque em custos é a contabilidade tradicional de custos. Essa visão de gerenciamento dos custos refletiu e reforçou, 
historicamente, a busca por uma alta eficiência em todos os recursos produtivos. Isso decorre do entendimento de que o melhor desempenho global da empresa é obtido a partir somatório dos ótimos locais, denominado Mundo dos Custos, onde a meta é a constante redução dos custos da empresa (GOLDRATT, 1992; LACERDA; CASSEL; RODRIGUES, 2010; COX; SCHLEIER JUNIOR, 2010). Esse é o mundo das variáveis independentes sugerido por Goldratt e Cox (2002). Na percepção do Sistema Toyota de Produção (OHNO, 1997) e da Teoria das Restrições (GOLDRATT, 1992), a contabilidade tradicional de custos deveria ser combatida, pois ela era a principal inimiga da produtividade. No Mundo dos Custos, os balizadores para a tomada de decisão são indicadores que apresentam grau de importância nesta ordem: i) Despesas Operacionais; ii) Inventário; e iii) Ganho (LACERDA; RODRIGUES, 2009). Esse modo de pensar está, ainda, fundamentado na ideia de que, ao se utilizarem todos os recursos ao máximo, a empresa estaria desfrutando completamente do investimento, gerando assim economia (SOUZA; PIRES, 1999). Por sua vez, o Mundo dos Ganhos (GOLDRATT, 1992) gradua diferentemente a importância dos mesmos indicadores. A ordem de importância é alterada para: i) Ganho; ii) Inventário; e iii) Despesas Operacionais (LACERDA; RODRIGUES, 2009). A partir da analogia de que a empresa é uma corrente, o desempenho do sistema é limitado pelo elo mais fraco, ou seja, a sua restrição (GOLDRATT; COX, 2002; COX; SCHLEIER JUNIOR, 2010). Portanto, a partir da visão de ganho e não de custo, a máxima utilização dos recursos é coerente nos recursos onde estão localizadas as restrições do sistema, pois são eles que limitam o ganho global da empresa (GOLDRATT; COX, 2002; LACERDA; RODRIGUES, 2009).

Assim, empresas, ao adotarem praticas associadas aos preceitos produção em massa e gestão baseada em indicadores do Mundo dos Custos, tendem a buscar a maximização do uso da capacidade em todos os recursos. Contudo, segundo El-Rayah (1979b), Smunt e Perkins (1985), Umble e Srikanth (1995), Goldratt e Cox (2002), o fato de uma linha de produção ou montagem, por exemplo, ser perfeitamente balanceada em termos de tempo médio de processamento de cada estação não é garantia de que o sistema irá operar com a máxima produtividade. Esse fato deve-se à maneira incremental de adição de capacidade e à influência da variabilidade e de eventos dependentes que estão presentes nesse processo (HOPP; SPEARMAN, 2001). Para Umble e Srikanth (1995) e Hopp e Spearman (2001), em geral, na manufatura é praticamente impossível obter um exato balanceamento da capacidade produtiva. Isso ocorre porque muitas resultantes do dimensionamento ideal da capacidade são frações, o que exige um arredondamento incremental dos valores calculados, uma vez que não há condições de se praticar, por exemplo, de 3,5 operadores (4 operadores) ou 5,7 máquinas (6 máquinas). Essa característica inviabiliza o efetivo balanceamento e pode contribuir para a geração de excesso de capacidade, impactando em aumento de estoques em processo e, por consequência, aumento das esperas e do lead time.

Nessa linha de discussão, o sistema de produção enxuta, ao buscar o nivelamento da carga de trabalho para atender o takt time (tempo disponível para trabalho dividido pela demanda do mercado), por meio do Heijunka Box, implica também no balanceamento de capacidade (ROTHER; SHOOK, 1998; WOMACK; JONES, 2004). Entretanto, a implantação desse sistema requer que um conjunto de pressupostos esteja presente no ambiente de produção, trazendo estabilidade para ele. Goldratt (2009) aponta três pressupostos que fundamentam a estabilidade estruturante do sistema de produção enxuta. Esses pressupostos são: a) estabilidade do ambiente, onde os processos e os produtos não se alteram significativamente em um período considerável de tempo; b) estabilidade na demanda dos produtos, ou seja, produzir frequentemente a gama de produtos definidos; c) estabilidade na carga total alocada aos vários recursos da manufatura, decorrentes dos pedidos postados pelo mercado. Os três pressupostos de estabilidade requeridos para a plena utilização do sistema de produção enxuta estão vinculados a elementos externos à organização, ou seja, relacionados ao projeto e comercialização de seus produtos e não com a lógica de produção (GOLDRATT, 2009).

Ademais, conforme salienta Goldratt (2009), nem todas as empresas inseridas no mercado desfrutam dessa condição de estabilidade. Assim sendo, pelo menos um aspecto da instabilidade, senão os três, está presente em parte significativa dos sistemas produtivos. Essa instabilidade evidencia-se com maior intensidade em produtos com ciclo de vida reduzido. Nesses casos, estocar pode representar obsolescência e longos lead times podem representar a redução do tempo disponível para a comercialização, ocasionando possíveis desabastecimentos e a não efetivação de vendas. Empresas que ofertam diversificado portfólio de produtos ao mercado também são vulneráveis à instabilidade, uma vez que a demanda varia e manter estoques de todos os itens onera a cadeia de suprimentos. Por fim, a instabilidade na carga de trabalho contribui para um baixo desempenho do nível de serviço das empresas (COX; SCHLEIER JUNIOR, 2010).

Procurando gerir a instabilidade no sistema produtivo, a Teoria das Restrições (Theory of Constraints - TOC) apresenta uma proposição de balanceamento do fluxo a partir da identificação das operações restritivas e da definição de um algoritmo de sequenciamento denominado Tambor-Pulmão-Corda 
(TPC). Nesse ínterim, a proposição de um modelo estratégico de gerenciamento da capacidade produtiva na manufatura integrando os conceitos da TOC e da Manutenção Produtiva Total pode ser encontrado no estudo de Pacheco et al. (2013), contribuindo para a gestão prática de sistemas produtivos. Pacheco, Jung e Caten (2013) analisaram os pontos de convergência e as divergências entre a Teoria das Restrições e o Seis Sigma quando usados de maneira integrada para a melhoria contínua operacional de sistemas de manufatura. Concluiu-se que a Teoria das Restrições e Seis Sigma possuem diversos elementos complementares que se sobrepõe aos pontos divergentes e que há um vasto campo de pesquisa a ser explorado sobre o tema. Junto a essas ações, a TOC definiu três tipos de capacidade, para absorver o impacto das flutuações estatísticas sobre o desempenho das restrições (GOLDRATT, 1992; COX; SCHLEIER JUNIOR, 2010). Estas capacidades são:

a) Capacidade produtiva: representa a capacidade que a empresa irá efetivamente usar do recurso para atender à demanda;

b) Capacidade protetiva: capacidade excessiva nos recursos não restritivos para que esses não interrompam o fluxo da operação restritiva e, consequentemente, o processo como um todo; c) Capacidade ociosa: entendida como a diferença entre a capacidade disponível e as capacidades produtivas e protetivas.

Dessa forma, o tamanho da capacidade protetiva varia diretamente em função da variabilidade (variação dos tempos de processo, nível de qualidade, paradas de máquinas etc.) e inversamente em relação à quantidade de estoque em processo. Ou seja, quanto maior o estoque em processo, menor pode ser essa capacidade (GOLDRATT, 1992; SOUZA; BAPTISTA, 2010; COX; SCHLEIER JUNIOR, 2010; DETTMER, 2000; CHAKRAVORTY; ATWATER, 2006). Todavia, o aumento do estoque em processo não elimina a capacidade protetiva, uma vez que para isso seria necessário estoque infinito (GOLDRATT, 1992).

Os pressupostos da TOC para absorver o efeito da variabilidade passam pelo posicionamento de pulmões e excedente de capacidade para proteger os recursos restritivos e, por consequência, o sistema produtivo. Outro pressuposto trata de elevados níveis de qualidade para os recursos à montante $\mathrm{e}$ à jusante da(s) operação(ões) restritiva(s), para não comprometer o ganho global do sistema. Segundo Goldratt e Cox (2002) e Umble e Srikanth (1995), o custo de manter um pulmão e a sobra de capacidade é compensado pelo aumento da produtividade do sistema, presumindo que os produtos fabricados estarão vendidos.

Segundo Goldratt e Cox (2002), Umble e Srikanth (1995), Jacob, Bergland e Cox (2010) e Cox e Schleier
Junior (2010), os efeitos indesejados que ocorrem na manufatura, tais como aumento de inventários e do lead time, redução da produtividade e atrasos na entrega estão relacionados com a variabilidade e as relações de interdependência existentes. Dependência está relacionada ao fato de que uma operação será realizada após o término da anterior. Dessa forma, determinadas operações iniciarão apenas quando as peças necessárias estiverem disponíveis. Essas peças, por sua vez, necessitam ser processadas por um determinado recurso, que novamente dependerá do abastecimento de material de outro recurso e assim por diante. Goldratt e Cox (2002) e Jacob, Bergland e Cox (2010) denominam esse fenômeno de eventos dependentes na manufatura. Para Souza e Pires (1999), outro tipo de dependência importante em ambientes fabris são as dependências decorrentes de interações entre produtos que disputam um mesmo recurso para serem produzidos, assim, elas estão estreitamente relacionadas à qualidade do sequenciamento das operações.

As flutuações estatísticas são eventos que não podem ser previstos e geram variabilidade na operação e, por consequência, no sistema (GOLDRATT, 1992; GOLDRATT; COX, 2002; JACOB; BERGLAND; COX, 2010; COX; SCHLEIER JUNIOR, 2010). Alguns exemplos típicos de flutuações estatísticas são: baixa qualidade de materiais comprados, quebra de máquinas, disponibilidade de ferramentas, absenteísmo, falta de material para processar no recurso, variações no comportamento, entre outros (COX; SCHLEIER JUNIOR, 2010). Essas flutuações estão presentes nos modelos SMS e MMS classificados por Ghosh e Gagnon (1989).

Segundo Souza e Pires (1999, p. 113),

[...] Ainda que para cada recurso, visto isoladamente, a soma de suas "flutuações estatísticas" tenda a zero, quando examinada a partir de uma cadeia sequencial de operações (eventos dependentes), cada variação acima da média em qualquer elemento do sistema tende a não ser absorvida pelo sistema como um todo, enquanto que as flutuações abaixo da média são propagadas ao longo da cadeia.

Dudley (1963), Smunt e Perkins (1985), Umble e Srikanth (1995), Hopp e Spearman (2001) apregoam que os tempos de operação são considerados variáveis aleatórias. Isso implica que esses tempos podem, portanto, ser expressos por uma distribuição probabilística.

Para ilustrar, didaticamente, o conteúdo exposto, a Tabela 1 expõe o impacto das variabilidades ou flutuações estatísticas e das relações de dependência na manufatura. Considerando um sistema com três recursos que possuem plena demanda e apresentam capacidades de processamento individuais iguais 
de $10 \pm 2$ peças por dia, ou seja, o sistema adota o balanceamento das capacidades, com todos os recursos nivelados, e considerando ainda que o produto em questão deve passar obrigatória e respectivamente pelos três recursos (A, B e C), ao final de 10 dias o sistema terá produzido 100 peças (10 peças em média por dia vezes 10 dias)? A resposta é não.

Esse exemplo retrata o efeito da variabilidade dos tempos de processamento e das relações de dependência entre os recursos. Ou seja, o acúmulo das flutuações estatísticas limita a produção da fábrica como um todo, até o menor resultado individual dos recursos. Assim, a produção real do sistema no final do período é menor, nesse caso, que a produção individual dos recursos. Ao balancear a capacidade, qualquer variabilidade que reduza o volume produzido impacta na redução do volume total do período, uma vez que há dependência entre as três operações.

Isso explica o resultado obtido na produção total do sistema ser de 89 itens e não de 100. Esse simples exemplo ilustra o impacto da variabilidade no processo, contribuindo para a necessidade de balancear o fluxo e não a capacidade, uma vez que são esses recursos restritivos que irão limitar o desempenho global do sistema. Outros exemplos semelhantes podem ser encontrados em Souza e Pires (1999), Umble e Srikanth (1995) e Hopp e Spearman (2001).

Para balancear o fluxo é necessária uma restrição que será o ponto focal do sistema, fazendo com que os demais recursos relacionados estejam subordinados a essa restrição (GOLDRATT, 1992; GOLDRATT; COX, 2002; JACOB; BERGLAND; COX, 2010; UMBLE; SRIKANTH, 1995).

O exemplo apresesentado na Tabela 1 pode ser reconstruído a partir da visão do balanceameto do fluxo, conforme a Tabela 2, onde o recurso B é definido como o gargalo. Uma vez que esse processo apresenta variabilidade, as capacidades dos recursos $\mathrm{A}$ e $\mathrm{C}$ são elevadas para $12 \pm 2$ e a capacidade de $C$ permanece em $10 \pm 2$, com o mesmo comportamento apresentado na Tabela 1. Com esse movimento percebe-se que a restrição, que antes migrava entre os recursos, agora se concentra no recurso B. A Tabela 2 ilustra esse comportamento.

Percebe-se, com o exemplo apresentado na Tabela 2, que mesmo com variabilidade a decisão de estabelecer a restrição em um dos recursos contribui para que ele não fique oscilando entre os recursos, como pode ser verificado na Tabela 1. Essa ação possibilitou que o sistema elevasse sua produção, produzindo agora 100 e não mais os 89 do primeiro exemplo. Dessa forma, na presença de variabilidade no processo produtivo, a ação de definir o recurso restritivo contribui para a focalização da gestão, impedindo que ela se disperse pelos recursos do sistema a cada momento.

Uma das vantagens de focalizar a gestão no gargalo, é que as ações de melhoria para elevar a capacidade do sistema são focadas em um ou poucos recursos. Um conjunto de estratégias para elevar a capacidade de sistemas produtivos foi apresentada em Pacheco et al. (2014).

A focalização no recurso restritivo possibilita que a empresa subordine os demais recursos a esse, caminhando no sentido da sincronização do fluxo dos materisis nesse sistema (GOLDRATT, 1992; GOLDRATT; COX, 2002; JACOB; BERGLAND; COX, 2010; UMBLE; SRIKANTH, 1995). Essa sincronização materializa-se pelo fluxo dos materias, a fim de atender a programação do recurso restritivo, fazendo coom que não faltem e sobrem materiais além do projetado. Comparando-se os dois sistema, aquele que for sincronizado apresentará um lead time menor, reduzidos estoques em processo e consequente redução e filas. Um fluxo de materiais não sincronizado

Tabela 1. Exemplo do efeito da variabilidade nos tempos de processamento - abordagem capacidade.

\begin{tabular}{ccccc}
\hline \multicolumn{5}{c}{ Capacidade diária por recurso } \\
\hline Dia & Recurso A & Recurso B & Recurso C & Produção total do sistema \\
\hline 1 & 11 & 10 & 8 & 8 \\
2 & 8 & 11 & 9 & 8 \\
3 & 9 & 12 & 10 & 9 \\
4 & 9 & 8 & 10 & 8 \\
5 & 11 & 10 & 11 & 10 \\
6 & 10 & 9 & 10 & 9 \\
7 & 11 & 10 & 12 & 10 \\
8 & 11 & 10 & 12 & 9 \\
9 & 10 & 12 & 9 & 8 \\
Total & 10 & 8 & 9 & $\mathbf{8 9}$ \\
Média & $\mathbf{1 0 0}$ & $\mathbf{1 0 0}$ & $\mathbf{1 0 0}$ & $\mathbf{8 , 9}$ \\
\hline
\end{tabular}


Tabela 2. Exemplo do efeito da variabilidade nos tempos de processamento - abordagem fluxo.

\begin{tabular}{ccccc}
\hline \multicolumn{5}{c}{ Capacidade diária por recurso } \\
\hline Dia & Recurso A & Recurso B & Recurso C & Produção total do sistema \\
\hline 1 & 11 & 10 & 12 & 10 \\
2 & 13 & 11 & 12 & 11 \\
3 & 14 & 12 & 13 & 12 \\
4 & 9 & 8 & 10 & 8 \\
5 & 11 & 10 & 11 & 10 \\
6 & 10 & 9 & 11 & 9 \\
7 & 11 & 10 & 12 & 10 \\
8 & 11 & 10 & 12 & 10 \\
9 & 13 & 12 & 14 & 8 \\
10 & 10 & 8 & 9 & $\mathbf{1 0 0}$ \\
Total & $\mathbf{1 1 3}$ & $\mathbf{1 0 0}$ & $\mathbf{1 1 6}$ & $\mathbf{1 0}$ \\
Média & $\mathbf{1 1 , 3}$ & $\mathbf{1 0}$ & $\mathbf{1 1 , 6}$ &
\end{tabular}

contribui para o baixo atendimento dos prazos de entrega e para a elevação do lead time, já que parte significativa desse tempo está relacionada a esperas a que os lotes são submetidos durante sua produção. Nesse aspecto, tanto a TOC quanto a Produção Enxuta buscam a sincronização visando a redução do lead time e de estoques, porém com ferramentas distintas, a TOC com o sistema Tambor-PulmãoCorda e a Produção Enxuta com o sistema Kanban. Na sequência são apresentados alguns conceitos associados ao pensamento sistêmico necessários para a compreensão do trabalho.

\section{Pensamento sistêmico}

A consciência de que o resultado dos processos deve prevalecer ao resultado das partes que o compõem, ou seja, que as melhorias nas operações devem ser subordinadas à melhoria do processo, é algo apregoado por diversos trabalhos (ANTUNES JÚNIOR et al., 2008; DENNIS, 2008; COX; SCHLEIER JUNIOR, 2010; GOLDRATT, 2009; SHINGO, 1996a, 1996b, 2010).

Essa percepção da importância de um elemento (operação) a partir do todo no qual ele está inserido (processo) é aderente aos preceitos do Pensamento Sistêmico. A ênfase nas partes é reconhecida como mecanicismo ou reducionismo, a ênfase no todo está vinculada a uma percepção holística, organísmica ou ecológica (CAPRA, 1996). Na ciência do século XX, a perspectiva holística passa a ser conhecida como sistêmica e a maneira de perceber as relações entre os distintos elementos formadores de um sistema passou a ser conhecida como Pensamento Sistêmico (SENGE, 2000).

Para Senge (2000), o Pensamento Sistêmico constituí uma das princiais disciplinas a serem incorporadas pelas organizações. Por disciplina,
Senge (2000) entende meio de que a organização se vale para adquirir habilidades importantes para seu desempenho ao longo do tempo. Andrade et al. (2006) complementam o entendimento ao definirem disciplina como o arcabouço de conhecimentos sistematizados que são relevantes para um fim específico. O Pensamento Sistêmico é definido por Andrade et al. (2006) como a competência de avaliar o impacto das decisões no tempo e no espaço.

Uma visão sistêmica compreende a percepção do impacto das ações no tempo e no espaço, entendendo os feedbacks repassados pelos elementos do sistema, suas relações circulares, bem como os atrasos (delays) existentes entre as ações e sua consequência mais ampla dentro do sistema (MORANDI et al., 2013). Segundo Senge (2000), o Pensamento Sistêmico têm sido aplicado para entender as relações entre variáveis e os impactos em uma ampla variedade de sistemas, a saber: empresariais, urbanos, regionais, econômicos, políticos, ecológicos e até mesmo fisiológicos. Senge (2000) reforça a importância do uso dessa disciplina, pois ela é fundamental para estabelecer uma nova mentalidade nos profissionais possibilitando, assim, a construção de organizações com capacidade de aprendizagem duradoura.

O Pensamento Sistêmico tem uma linguagem própria que busca evidenciar o sentido da influência entre as variáveis (independente e dependente ou de causa e efeito), que por sua vez podem ser de natureza direta ou inversa. Quando ocorre uma variação em uma variável independente e gera-se uma variação no mesmo sentido na variável dependente (quanto mais A, mais B, ou quanto menos A, menos B), diz-se que essa relação é diretamente proporcional. Contudo, quando ocorre uma variação na variável independente e gera-se uma variação em sentido oposto na variável dependente (quanto mais $\mathrm{A}$, menos $\mathrm{B}$, ou 
quanto menos A, mais B), diz-se que essa relação é inversamente proporcional.

A Figura 1 traz exemplos desses dois tipos de influência (direta e inversamente proporcional), a partir da utilização da notação sistêmica para a releitura de uma linguagem do cotitiano.

A linguagem sistêmica se expressa em distintas notações, assim, para a confecção das estruturas sistêmicas deste trabalho foi utilizada a notação proposta por Morandi et al. (2013), expressa na Figura 1: setas contínuas $=$ relação direta e setas pontilhadas $=$ relações inversas .

O Pensamento Sistêmico neste estudo é utilizado para identificar as relações circulares (feedback) entre o conjunto de elementos suportados em cada uma das abordagens de balanceamento. Além disso, apoia a compreenção do impacto dessa relação no tempo e no espaço. A seguir expõem -se a análise sistêmica e a discussão entre o balanceamento do fluxo e o balanceamento da capacidade.

\section{Análise sistêmica e discussão crítica entre balanceamento de fluxo e de capacidade}

Parece ser possível construir uma visão geral que contenha alguns elementos que estão presentes nas abordagens do balanceamento de fluxo e do balanceamento da capacidade. Esses elementos são apresentados no Quadro 1.

Na sequência, esses pressupostos foram analisados e relacionados, cada abordagem em uma estrutura sistêmica. Essa etapa teve por objetivo visualizar as relações existentes entre eles e com outros elementos integrantes de um sistema produtivo. Além disso, buscou-se verificar as circularidades existentes nas relações de efeito-causa-efeito. A Figura 2 apresenta a estrutura sistêmica formada pelos pressupostos e outros elementos relacionados à abordagem do balanceamento da capacidade que reforçam a sua utilização.

Analisando as relações apresentadas na estrutura percebe-se que o volume de produção dos recursos é um importante fator na manutenção desse sistema. Esse volume resultante da máxima produção dos recursos contribui diretamente (seta contínua) para o aumento dos estoques (E1). Por consequência, contribui para o aumento da independência entre esses recursos, uma vez que há material suficiente para o abastecimento, contribuindo assim para o aumento da utilização e maior capacidade. Essa relação apresenta um enlace reforçador, em que mais produção representa mais eficiência que resulta em mais produção.

Uma segunda contribuição do volume de produção é representada pela relação inversa (seta pontilhada) com o custo unitário (E2), ou seja, quanto maior a produção, menor o custo unitário. As relações decorrentes da redução do custo unitário conduzem para a redução do custo total e consequente aumento do resultado da empresa. Essas relações resultam em maiores investimentos e na realização de ações de melhoria, que contribuem para reduzir a variabilidade em todos os recursos e, por consequência, para o aumento do volume de produção. Nesse segundo enlace também é verificada a presença de uma relação reforçadora, à medida que maior produção reflete-se em mais investimentos, que acabam reduzindo a variabilidade, com efeito de possibilitar maior produção.

A análise dessas relações resulta em um posicionamento crítico em relação à adoção dessa abordagem no que tange aos dois efeitos apresentados a partir do aumento do volume de produção (estoque em processo e custo unitário). Ao se aumentar os estoques em processo, aumentam as filas, que por sua vez trazem consigo o aumento dos tempos de espera dos lotes. É sabido que quanto mais tempo um lote espera para ser processado, maior será o seu tempo de atravessamento (lead time) dentro

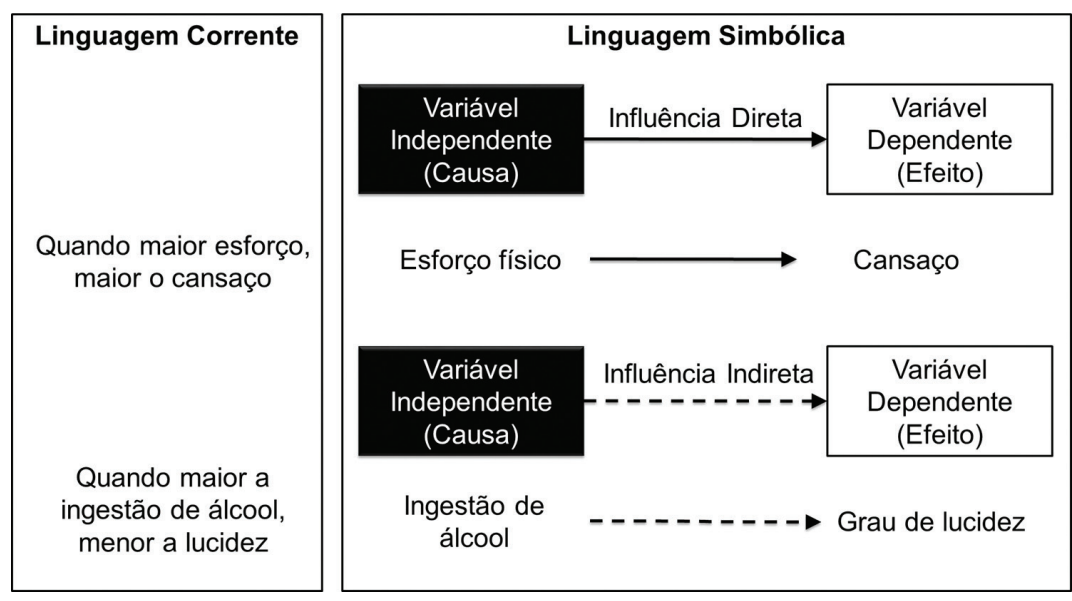

Figura 1. Relação entre as variáveis. Fonte: adaptado de Andrade et al. (2006). 
Quadro 1. Pressupostos das abordagens de balanceamento de fluxo e decapacidade.

\begin{tabular}{|l|l|}
\hline \multicolumn{1}{|c|}{ Elementos do balanceamento da capacidade } & \multicolumn{1}{c|}{ Elementos do balanceamento de fluxo } \\
\hline Alocar carga de acordo com a capacidade dos recursos. & $\begin{array}{l}\text { Alocar carga dos recursos não restritivos de acordo com } \\
\text { a necessidade do recurso restritivo. }\end{array}$ \\
\hline Maximizar a utilização dos recursos. & $\begin{array}{l}\text { Distinguir utilização e ativação dos recursos não } \\
\text { restritivos. }\end{array}$ \\
\hline Maximizar a eficiência dos recursos (ótimo local). & $\begin{array}{l}\text { Maximizar a eficiência no recurso que restringe o } \\
\text { melhor desempenho do sistema (ótimo global). }\end{array}$ \\
\hline $\begin{array}{l}\text { Reduzir as capacidades em excesso dos recursos, } \\
\text { ajustando-se à demanda. }\end{array}$ & $\begin{array}{l}\text { Tolerar capacidades em excesso nos recursos não } \\
\text { restritivos, visando obter a máxima produtividade no } \\
\text { recurso restritivo. }\end{array}$ \\
\hline $\begin{array}{l}\text { Garantir o suprimento dos recursos mantendo estoques } \\
\text { de materiais. }\end{array}$ & $\begin{array}{l}\text { Definir o volume e a variedade de materiais necessários } \\
\text { para garantir um determinado tempo de proteção de } \\
\text { funcionamento do recurso restritivo (pulmão tempo). }\end{array}$ \\
\hline $\begin{array}{l}\text { Focar as ações nos recursos que não estão atingindo suas } \\
\text { capacidades máximas. }\end{array}$ & Focar as ações no recurso restritivo. \\
\hline $\begin{array}{l}\text { Focar na redução dos custos por unidade (Mundo dos } \\
\text { Custos). }\end{array}$ & $\begin{array}{l}\text { Focar no aumento dos ganhos do sistema (Mundo dos } \\
\text { Ganhos). }\end{array}$ \\
\hline
\end{tabular}

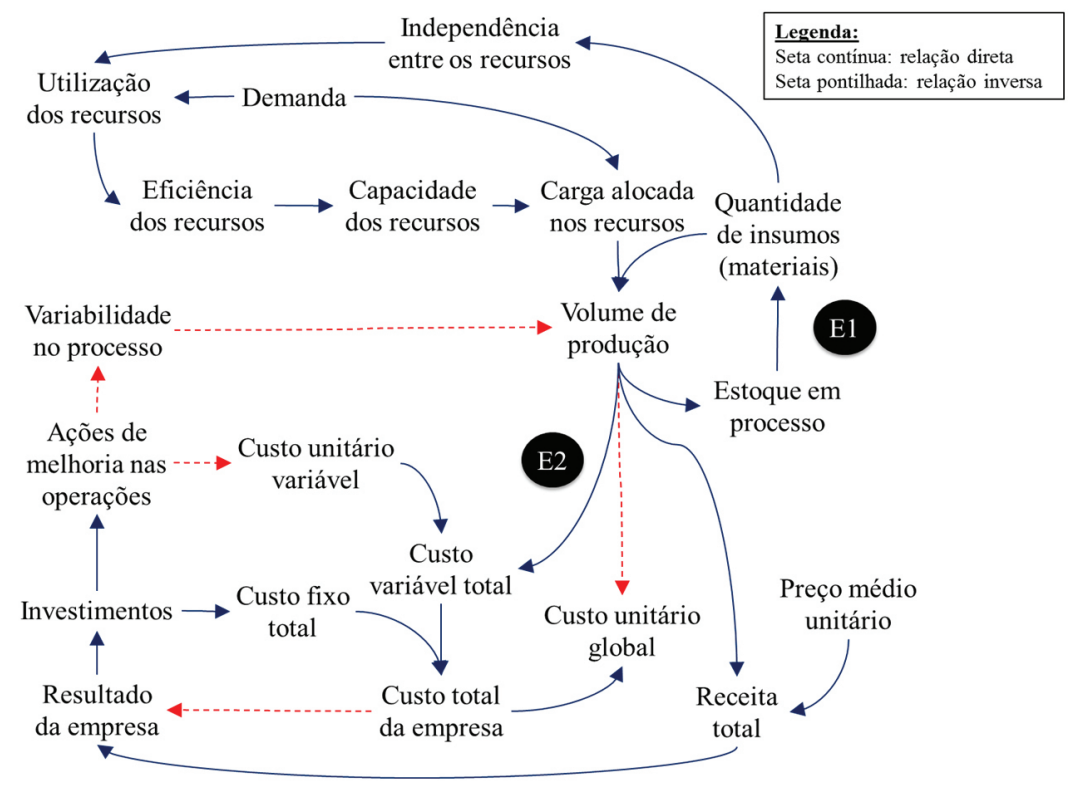

Figura 2. Estrutura sistêmica do balanceamento da capacidade.

do sistema produtivo. Essa ação pode representar atrasos na entrega do que foi contratado ou o não fechamento de pedidos devido ao longo prazo de entrega. $\mathrm{O}$ segundo efeito é a percepção de que o aumento do volume representa a redução dos custos unitários, uma vez que o valor dos custos fixos são rateados por um maior volume de produtos. Essa percepção encontra uma restrição, uma vez que esses produtos não são comercializados. Ou seja, os custos fixos serão desembolsados, mas a receita referente ao volume total de produção não será efetivada, assim os custos unitários não foram reduzidos. A variabilidade no processo exerce papel chave, pois é ela que contribui decisivamente para a manutenção da capacidade produtiva do sistema.

$\mathrm{Na}$ sequência dessa pesquisa foi elaborada a estrutura sistêmica, contendo os pressupostos e outros elementos de ligação, relacionada à abordagem do balanceamento do fluxo. A Figura 3 apresenta a estrutura em questão.

Ao se analisar a estrutura sistêmica referente à abordagem do balanceamento do fluxo percebe-se o papel central da identificação dos recursos restritivos, ou seja, aqueles que possuem capacidade inferior à demanda que recai sobre eles. A relação entre capacidade e demanda resulta em uma variável identificada na estrutura como restrições 


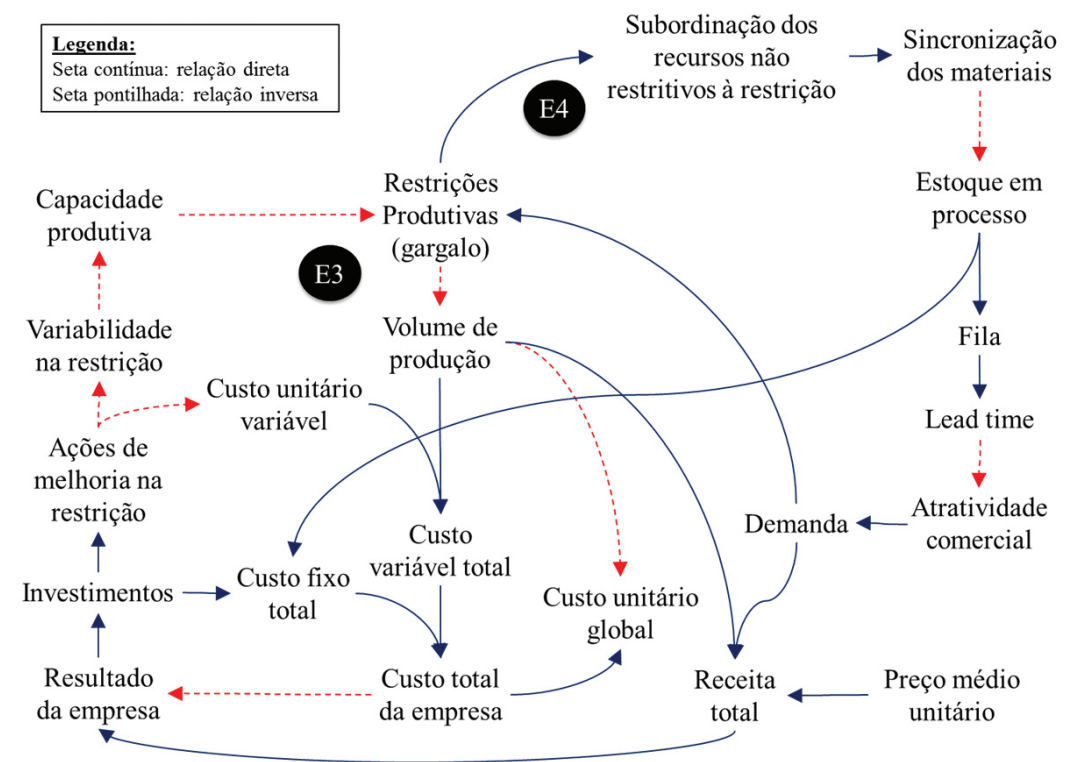

Figura 3. Estrutura sistêmica: balanceamento do fluxo.

produtivas - gargalo. A leitura dessa relação pode ser assim realizada: a) quanto mais capacidade, menos restrições produtivas (relação inversa, seta pontilhada); b) quanto mais demanda, mantendo-se a capacidade estável, mais restrições produtivas (relação direta, seta contínua). Esses recursos restritivos definem duas prioridades do sistema: o volume a ser produzido e a forma como serão programados os recursos não restritivos (subordinação à restrição).

Por um lado, quanto maior o volume produzido pelo sistema (E3), maior é seu custo variável total, que somado ao custo fixo total resulta no custo total da empresa. Por outro lado, quanto maior o volume produzido e maior a demanda e o preço, maior é a receita total da empresa. Assim, a relação entre receita e custo define o resultado da empresa. O resultado positivo contribui para a realização de investimentos em ações de redução da variabilidade. Aqui há uma diferença em relação à estrutura do balanceamento da capacidade, pois as ações de redução e variabilidade agora são focadas aos recursos restritivos, uma vez que são eles que limitam a capacidade produtiva do sistema. O segundo enlace a partir das restrições do sistema (E4) traz a relação de subordinação dos recursos não restritivos ao gargalo. Quanto maior a subordinação à restrição, maior é a sincronização e a consequente redução dos estoques em processo. A redução dos estoques reduz as filas, que reduzem o lead time, impactando no aumento da atratividade comercial (aumento do tempo de resposta ao mercado). Essa atratividade é ponto chave para o aumento da demanda do mercado pelos produtos da empresa. Assim, com o aumento da demanda a restrição retorna novamente para dentro da empresa e não se desloca para o mercado, sendo tratada conforme apresentado no enlace E3 da Figura 3.

A partir do entendimento proposto pelas estruturas sistêmicas é possível perceber que a estabilidade do processo, ou seja, redução da variabilidade, é um ponto determinante na adoção de uma ou outra abordagem. Nesse ponto da investigação emerge o seguinte questionamento: até que ponto os esforços de balancear as capacidades contribuem mais para a redução de custo do que os esforços de melhoria e da estabilidade do sistema?

Assim, processos com maior variabilidade poderiam caminhar no sentido de adotar uma abordagem de balanceamento do fluxo. Por sua vez, processos com menor variabilidade poderiam adotar a abordagem do balanceamento da capacidade.

Portanto, a fim de não comprometer o ganho da organização, na lógica do balanceamento do fluxo, é relevante haver esforços para reduzir a variabilidade na restrição. Ao balancear a capacidade e buscar a máxima utilização dos recursos produtivos há geração de estoques em processo e, por consequência, aumento das filas, o que acarreta aumento do lead time. Por sua vez, o balanceamento do fluxo, a partir da restrição, leva a uma redução dos estoques em processo, visto que esses serão projetados para proteger somente o gargalo. Ao reduzirem-se os estoques em processo há também a redução das filas e, com efeito, redução do lead time. Portanto, ações práticas no sentido de mitigar os agentes que desaceleram o processo são desejáveis na manufatura.

$\mathrm{O}$ adequado balanceamento do fluxo também contribui na programação logística, uma vez que focaliza as ações na restrição, contribuindo 
para a acuracidade da programação. Contudo, o balanceamento do fluxo pela restrição demanda o correto dimensionamento matemático da folga de capacidade dos recursos não restritivos e da proteção do sistema. Nesse ponto, uma heurística voltada para o entendimento do comportamento da demanda do mercado pode contribuir de maneira significativa para que o dimensionamento das folgas dos recursos não restritivos e o dimensionamento dos pulmões de proteção do ganho sejam confiáveis. Da mesma maneira, dado que o sistema será balanceado pelo fluxo, a partir da restrição, os esforços de melhoria da qualidade e redução da variabilidade devem estar focados nele.

É possível estabelecer uma convergência entre a abordagem do balanceamento de fluxo e de capacidade. A alternativa de convergência entre as duas abordagens é uma possibilidade, ou seja: priorizar os esforços claramente na restrição, mantendo ações nos demais recursos, à montante e à jusante do gargalo, condicionadas ao mesmo. O resultado esperado com essa articulação deve impactar na redução do lead time e da despesa operacional e contribuirá para aumentar o ganho global.

Outro ponto a ser salientado diz respeito à redução da variabilidade nos demais recursos. Essa ação contribui para que a restrição esteja definida e sob controle, facilitando o encaminhamento de ações de melhoria contínua para elevar a eficiência e decorrente capacidade. Entretanto, ao longo do programa de ações de melhoria no gargalo devem-se monitorar os demais recursos a fim de identificar onde localiza-se a próxima restrição. Isso faz com que ações e investimentos similares possam ser compartilhadas entre os recursos.

A sugestão hipotética é que o gargalo seja elevado ao mesmo tempo em que gera-se folga de capacidade nos recursos não gargalos. Dessa forma, o gargalo permanecerá na mesma posição dentro dos sistemas, simplificando o gerenciamento da manufatura.

Assim, ações para realizar o correto planejamento da manufatura (causa) devem ser priorizadas, pois provocam impacto direto e indireto na redução do lead time (efeito), dado que ele é consequência desse planejamento.

\section{Considerações finais}

Este artigo busca estimular a discussão entre o uso das duas abordagens de balanceamento (capacidade e fluxo), bem como perceber as relações envolvidas, a partir de uma perspectiva sistêmica. A análise sistêmica contribui conceitualmente para evidenciar as convergências e divergências entre balancear o fluxo ou a capacidade, bem como as respectivas consequências para os sistemas de manufatura. As principais convergências encontradas foram: a importância da qualidade dentro da manufatura, o impacto da variabilidade e a necessidade de redução do lead time.

Quanto à decisão que prevalece no quotidiano dos engenheiros e gestores de manufatura entre balancear o fluxo ou a capacidade, o produto desta pesquisa sugere que o balanceamento do fluxo é superior ao balanceamento da capacidade em termos da otimização do desempenho da manufatura em relação aos aspectos de qualidade, variabilidade e velocidade.

Os principais fatores que sustentam esse argumento são: i) a restrição define o ganho global da manufatura (reduz ação das flutuações estatísticas e dependência); ii) balancear o fluxo a partir da restrição simplifica o planejamento da fábrica (evitando a busca de algoritmos complexos e imprecisos de balanceamento); iii) a priorização das ações de melhoria na variabilidade, qualidade e investimentos em geral se tornam mais claras a partir da visão da restrição do sistema.

Contudo, para sustentar o balanceamento de fluxo, sugerem-se algumas ações práticas que podem ser adotadas pelas áreas de gestão da manufatura. A primeira é o uso de uma boa heurística, voltada para entender o comportamento da demanda e dimensionar corretamente as folgas dos recursos não gargalos e as capacidades produtivas e protetivas do sistema, bem como planejar e programar a produção a partir do gargalo. E como ponto principal de alavancagem do balanceamento do fluxo, a presente pesquisa permitiu evidenciar que se devem priorizar claramente os esforços de melhoria da qualidade e redução da variabilidade no gargalo, mantendo ações paralelas nos demais recursos dispostos à montante e à jusante do gargalo, condicionadas ao mesmo.

Adotar decisões gerenciais que considerem o balanceamento de fluxo combinado com determinadas práticas típicas do balanceamento de capacidade, conforme aqui discutido, podem contribuir para elevar o ganho global da empresa.

Como forma de continuar as discussões realizadas neste trabalho, duas alternativas são indicadas. A primeira pauta-se na tentativa de preencher as lacunas que emergiram durante a pesquisa, tais como pesquisas que considerem as implicações e extensão da TOC ao se considerar o fator humano, não só na manufatura como também nas diversas áreas da empresa. Outro caminho de extensão de pesquisa é tentar evidenciar empiricamente sistemas produtivos que adotem a abordagem balanceamento de fluxo e balanceamento da capacidade, comparando o desempenho de indicadores como produtividade, tempo de atravessamento e ganho.

\section{Agradecimentos}

Os autores deste trabalho agradecem as importantes contribuições dos dois revisores durante o processo de avaliação. Os autores também agradecem ao Prof. 
PhD. Luis Henrique Rodrigues (PPGEPS/UNISINOS) pela contribuição na construção deste estudo. Também agradecemos ao suporte proporcionado pela Capes aos bolsistas de doutorado Prof. Diego Pacheco e Prof. Secundino Luis Henrique Corcini Neto e ao CNPq, pela concessão de Bolsa de Produtividade em Desenvolvimento Tecnológico e Extensão Inovadora (DTI2) ao Prof. Dr. Daniel Pacheco Lacerda.

\section{Referências}

ANDRADE, A. L. et al. Pensamento Sistêmico: Caderno de campo: o desafio da mudança sustentada nas organizações e na sociedade. Porto Alegre. Bookman, 2006.

ANTUNES JÚNIOR, J. A. V. et al. Sistemas de produção: conceitos e práticas para projeto e gestão da produção enxuta. Porto Alegre: Bookmann, 2008.

ANTUNES JUNIOR, J. A. V. Em direção a uma teoria geral do processo na administração da produção: uma discussão sobre a possibilidade de unificação da teoria das restrições e a teoria que sustenta a construção dos sistemas de produção com estoque zero. 1998. Tese (Doutorado em Administração)-Escola de Administração, Universidade Federal do Rio Grande do Sul, Porto Alegre, 1998.

ATWATER, J. B.; CHAKRAVORTY, S. S. Does protective capacity assist managers in competing along time-based dimensions? Production and Inventory Management Journal, v. 35, p. 53-59, 1996.

BECKER, C.; SCHOLL, A. A survey on problems and methods in generalized assembly line balancing. European Journal of Operational Research, v. 168, p. 694-715, 2006. http://dx.doi.org/10.1016/j. ejor.2004.07.023

BOYSEN, N.; FLIEDNER, M; SCHOLL, A. A classification of assembly line balancing problems. European Journal of Operational Research, v. 183, p. 674-693, 2007. http://dx.doi.org/10.1016/j.ejor.2006.10.010

CAMARGO, E. A.; PACHECO, D. A. J. Aplicando o balanceamento de operações para melhorar o desempenho da indústria. Espacios (Caracas), v. 34, p. 1-15, 2013.

CAPRA, F. A teia da Vida: Uma nova compreensão cientifica dos sistemas vivos. 11. ed. São Paulo: Editora Cultrix, 1996.

CHAKRAVORTY, S. S.; ATWATER, J. B. Bottleneck management: theory and practice. Production Planning \& Control, v.17, n. 5, p. 441-447, 2006. http://dx.doi. org/10.1080/09537280600682752

COX, J. F.; SCHLEIER JUNIOR, J. G. Theory of Constraints: Handbook. New York: McGraw-Hill, 2010.

DAVIS, L. E. Pacing effects on manned assembly lines. International Journal of Production Research, v. 4, n. 3, p. 171-184, 1966. http://dx.doi. org/10.1080/00207546508919974

DENNIS, P. Produção Lean Simplificada: Um guia para entender o Sistema de produção mais poderoso do mundo. Porto Alegre: Bookman, 2008.

DETTMER, W. Beyond Lean Manufacturing: Combining Lean and the Theory of Constraints for Higher Performance. Port Angeles, 2000. Disponível em: <http://
www.goalsys.com/books/documents/TOCandLeanPaperrev.1.pdf>. Acesso em: 21 mar. 2013.

DUDLEY, N. A. Work time distribuitions. International. Journal of Production Research, v. 2, p. 137, 1963. http://dx.doi.org/10.1080/00207546308947819

EL-RAYAH, T. E. The effect of inequality of interstage buffer capacities and operations time variability on the efficiency of production line systems. International Journal of Production Research, v. 17, n. 1, p. 77-89, 1979a. http://dx.doi.org/10.1080/00207547908919596

EL-RAYAH, T. E. The efficiency of balanced and unbalanced production lines. International Journal of Production Research, v. 17, n. 1, p. 61-75, 1979b. http://dx.doi. org/10.1080/00207547908919595

FRY, T. D.; RUSSELL, G. R. Capacity allocation strategies in a hypothetical job-shop. International Journal of Production Research, v. 31, n. 5, p. 1097-1115, 1993. http://dx.doi.org/10.1080/00207549308956777

GUERREIRO, R. A teoria das restrições e o sistema de gestão econômica: Uma proposta de Integração Conceitual. 1995. Tese (Livre-Docência)-Faculdade de Econômica, Administração e Contabilidade, Universidade de São Paulo, São Paulo, 1995.

GHOSH, S.; GAGNON, R. A comprehensive literature review and analysis of the design, balancing and scheduling of assembly systems. International Journal of Production Research, v. 27, n. 4, p. 637-670, 1989. http://dx.doi.org/10.1080/00207548908942574

GOLDRATT, E. M.; COX, J. A meta: um processo de aprimoramento contínuo. São Paulo: Nobel, 2002.

GOLDRATT, E. M. A Síndrome do Palheiro. São Paulo: IMAM, 1992.

GOLDRATT, E. M. Standing on the Shoulders of Giants: Production concepts versus production applications The Hitachi Tool Engineering example. Gestão e Produção, v. 16, n. 3, p. 333-343, 2009. http://dx.doi.org/10.1590/ S0104-530X2009000300002

GOUGH, D.; OLIVER, S.; THOMAS, J. An Introduction to Systematic Reviews. London: Sage Publications Ltd, 2012. 304 p.

HAYES, R.; WHEELWRIGHT, C. Link manufacturing process and product life cycles. Harvard Business Review, v. 57, p. 33-140, 1979.

HILLIER, F. S.; BOLING, R. W. The effect of some design factors on the efficiency of production lines with variable operations times. Journal of Industrial Engineering, v. 17, n. 12, p. 651-658, 1966.

HOPP, W.; SPEARMAN, M. L. Factory Physics. Boston: Irwin, 2001.

JACOB, D.; BERGLAND, S.; COX, J. Velocity: Combining Lean, Six Sigma, and the Theory of Constraints to Achieve Breakthrough Performance: A Business Novel. Hardcover. 2010.

KRIENGKORAKOT, N.; PIANTHONG, N. The Assembly Line Balancing Problem: Review articles. KKU Engineering Journal, v. 34, n. 2, p. 133-140, 2007.

LACERDA, D.; RODRIGUES, L. H. Uma Discussão sobre o Mundo dos Custos e o Mundo dos Ganhos sob o Ponto de Vista da Teoria das Restrições. Sistemas e Gestão, v. 4, n. 1, p. 36-54, jan./abr. 2009. 
LACERDA, D. P.; CASSEL, R. A.; RODRIGUES, L. H. Service process analysis using process engineering and the theory of constraints thinking process. Business Process Management Journal, v. 16, n. 2, p. 264-281, 2010. http://dx.doi.org/10.1108/14637151011035598

LITTElL, J. H.; CORCORAN, J.; PILlAI, V. K. Systematic reviews and meta-analysis. New York: Oxford University Press, 2008

MILTENBURG, J. Setting manufacturing strategy for a factory-within-a-factory. International Journal of Production Economics, v. 113, p. 307-323, 2008. http://dx.doi.org/10.1016/j.ijpe.2007.09.001

MORANDI, M. I. W. M. et al. Foreseeing Iron Ore Prices Using System Thinking and Scenario Planning. Systemic Practice and Action Research, p. 1-37, 2013. 10.1007/ s11213-013-9277-9 http://dx.doi.org/10.1007/ s11213-013-9277-9

NAKAJIMA, S. Introduction to TPM - Total Productive Maintenance. Cambridge: Productivity Press, 1988.

OHNO, T. O Sistema Toyota de Produção: além da produção em larga escala. Porto Alegre: Bookman, 1997.

OZPEYNIRCI, S.; AZIZOGLU, M. Capacity allocation problem in flexible manufacturing systems: branch and bound based approaches. International Journal of Production Research, v. 47, n. 21, p. 5941-5958, 2009.

PACHECO, D. A. J. et al. The proposition of a model of management in manufacturing capacity. Espacios (Caracas), v. 34, p. 1-14, 2013.

PACHECO, D. A. J.; JUNG, C. F.; CATEN, C. S. T. Teoria das Restrições e Seis Sigma: limites e possibilidades de integração para a melhoria contínua. Espacios (Caracas), v. 34, p. 1-8, 2013.

PACHECO, D. A. J. et al. Strategies for increasing productivity in production systems. Independent Journal of Management \& Production, v. 5, p. 1-16, 2014.

PACHECO, D. A. J. Integrando a Estratégia de Produção com a Teoria das Restrições, Lean e Seis Sigma: uma abordagem metodológica. 2012. Dissertação (Mestrado em Engenharia de Produção e Sistemas)-Universidade do Vale do Rio dos Sinos, São Leopoldo, 2012.

PACHECO, D. A. J. Teoria das Restrições, Lean Manufacturing e Seis Sigma: limites e possibilidades de integração. Production, 2014. Ahead to print.

PAYNE, S.; SLACK, N.; WILD, R. A note on the operating characteristics of balanced and unbalanced production flow lines. International Journal of Production Research, v. 10, n. 1, p. 93-98, 1972. http://dx.doi. org/10.1080/00207547208929908

PIKE, R.; MARTIN, G. E. The bowl phenomenon in unpaced lines. International Journal of Production Research, v. 32, p. 483-499, 1994

ROTHER, M.; SHOOK, J. Aprendendo a Enxergar: Mapeamento do Fluxo de Valor para adicionar valor e eliminar os desperdícios. São Paulo: Lean Institute Brasil, 1998.
SARKER, B. R. Some comparative and design aspects of series production systems. IIE Transactions, v. 16, p. 229-239, 1984. http://dx.doi. org/10.1080/07408178408974689

SCHOLL, A.; BECKER, C. State-of-the-art exact and heuristic solution procedures for simple assembly line balancing. European Journal of Operational Research, v. 168, p. 666-693, 2006. http://dx.doi.org/10.1016/j. ejor.2004.07.022

SENGE, P. M. A Quinta Disciplina: Arte, Teoria e Prática da Organização de Aprendizagem. São Paulo: Best Seller, 2000.

SHINGO, S. Kaizen e a arte do pensamento criativo. Porto Alegre: Bookman, 2010.

SHINGO, S. O Sistema Toyota de Produção do ponto de vista da Engenharia de Produção. Porto Alegre: Bookman, 1996a.

SHINGO, S. Sistema de produção com estoque zero: $\mathrm{O}$ Sistema Shingo para melhorias contínuas. Porto Alegre: Bookman, 1996b.

SKINNER, W. Manufacturing strategy: The story of its evolution. Journal of Operations Management, v. 25, p. 328-335, 2007. http://dx.doi.org/10.1016/j. jom.2006.10.008

SKINNER, W. Manufacturing: missing link in corporate strategy. Harvard Business Review, v. 47 p. 136-145, 1969.

SKINNER, W. The focused factory. Harvard Business Review, v. 52, p. 113-121, 1974.

SLACK, N.; CHAMBERS, S.; JOHNSTON, R. Administração da Produção. 3. ed. Atlas, 2009.

SMUNT, T. L.; PERKINS, W. C. Stochastic unpaced line design: review and further experimental results. Journal of Operations Management, v. 5, n. 3, p. 351-373, 1985. http://dx.doi.org/10.1016/0272-6963(85)90019-1

SOUZA, B. S.; BAPTISTA, H. R. Proposta de avanço para o método Tambor-Pulmão-Corda Simplificado aplicado em ambientes de produção sob encomenda. Gestão e Produção, v. 17, n. 4, p. 735-746, 2010. http://dx.doi. org/10.1590/S0104-530X2010000400008

SOUZA, F. B.; RENTES, A. F.; AGOSTINHO, L. O. A Interdependência entre controles de produção e critérios de alocação de capacidades. Gestão e Produção, v. 9, n. 2, p. 215-234, ago. 2002.

SOUZA, F. B.; PIRES, R. I. S. Análise e proposições sobre o balanceamento e uso de excesso de capacidade em recursos produtivos. Gestão e Produção, v. 6, n. 2, p. 111-126, ago. 1999.

UMBLE, M. M.; SRIKANTH, M. L. Synchronous Manufacturing: principles for world class excellence. Cincinnati: South-Western, 1995.

WOMACK, J. P.; JONES, D. A Mentalidade Enxuta. Editora Campus, 2004. 